\title{
Adjunctive resectional lung surgery for extensively drug-resistant tuberculosis
}

\author{
G. Dravniece*, K.P. Cain\# ${ }^{\#}$ T.H. Holtz ${ }^{\#}$, V. Riekstina*, V. Leimane* and R. Zaleskis
}

\begin{abstract}
Extensively drug-resistant (XDR) tuberculosis (TB) poses significant management challenges as there are limited pharmacological treatment options for cure. Adjunctive resectional lung surgery decreases case-fatality rates for some patients with multidrug-resistant tuberculosis (MDR-TB), but its use has not been well documented for patients with XDR-TB.

We describe 17 XDR-TB patients treated with surgery as part of their case management in Latvia during 1999-2005. One patient had no previous TB treatment history, 10 were previously treated for drug-susceptible TB and six were previously treated for MDR-TB. Mycobacterium tuberculosis isolates from the 17 patients were resistant to a mean of 9.2 drugs. Due to failure of pharmacological therapy, one due to a large cavity and one due to pulmonary haemorrhage, 15 patients were treated with surgery. Despite failure of pharmacological treatment in 15 out of 17 patients, eight (47\%) were cured with adjunctive surgical treatment. Surgery should be explored as a possible treatment option for patients with XDR-TB.
\end{abstract}

KEYWORDS: Epidemiology, extensively drug-resistant tuberculosis, mortality, multidrugresistant, surgery, tuberculosis

xtensively drug-resistant (XDR) tuberculosis (TB), defined as TB caused by Mycobacterium tuberculosis with resistance to at least isoniazid, rifampicin, a fluoroquinolone and one second-line injectable drug (capreomycin, kanamycin or amikacin) [1], is now recognised to exist worldwide, even in patients with no previous TB treatment history, and to commonly result in death [2-4]. Although $\sim 60-$ $75 \%$ of patients with multidrug-resistant (MDR) $\mathrm{TB}$, or persons with resistance to isoniazid and rifampicin, are cured with appropriate treatment in programmatic settings [5], preliminary results from Latvia show that just $30-40 \%$ of XDR-TB patients are cured [2]. Similarly, among XDR-TB patients treated in Estonia, Germany, Russia and Italy, just 39\% were cured [3]. These low cure rates are similar to those reported for TB treatment prior to the availability of effective anti-TB drugs [6].

There are currently limited data regarding optimal treatment strategies in patients with XDR-TB. Resectional lung surgery, in which a portion of lung with a high burden of $\mathrm{TB}$ is removed, is one treatment option that has been successfully used in addition to pharmacological therapy in MDR-TB patients [7, 8]. The probable mechanism by which surgery is useful is a decrease in the bacillary burden through removal of a large area of abnormal lung tissue and granulomatous cavities, where the penetration of drugs is typically poor. Such adjunctive surgical treatment might be useful in the treatment of XDR-TB; however, this is not known.

Latvia has had an active MDR-TB treatment programme since 1997 [9]. Adjunctive resectional lung surgery has been used for some patients with MDR-TB since the 1990s in Latvia. Some patients have XDR-TB, and a subset are treated with surgery. All decisions about treatment (including surgery) are made by a group of specialists at Latvia's national TB hospital (the State Agency of Tuberculosis and Lung Diseases, Riga, Latvia). The current report describes the present authors' experience in treating XDR-TB patients in Latvia with adjunctive resectional lung surgery in order to evaluate the role of surgery in XDR-TB treatment.

\section{METHODS}

A case series of patients who were diagnosed with MDR-TB in Latvia was conducted between January 1, 2000 and December 31, 2003. They met current criteria for XDR-TB and were treated with adjunctive resectional lung surgery.

The Latvian TB programme registers all TB patients in the country in the national TB registry. Details of their approach to the diagnosis and treatment of MDR- and XDR-TB have been previously reported [10]. Briefly, all TB patients have sputum specimens submitted for $M$. tuberculosis culture, identification and drug susceptibility
AFFILIATIONS

*Latvia State Agency of Tuberculosis and Lung Diseases, Riga, Latvia.

\#Division of Tuberculosis Elimination, Centers for Disease Control and Prevention, Atlanta, GA, USA.

"World Health Organization, European Regional Office, Copenhagen, Denmark

\section{CORRESPONDENCE}

G. Dravniece

State Agency of Tuberculosis and Lung Diseases p/o Cekule, Stopini Riga District

Latvia, LV2118

E-mail: gdravniece@tuberculosis.Iv

Received:

March 262008

Accepted after revision:

Jan 062009 
testing (DST) to first-line drugs (isoniazid, rifampicin, streptomycin, and pyrazinamide) at the time of diagnosis. For all patients with MDR-TB, sputum smear and culture tests are repeated monthly throughout treatment. All MDR-TB isolates are also tested for resistance to second-line drugs, including kanamycin, capreomycin, ofloxacin, protionamide, cycloserine, $p$-aminosalicylic acid and thioacetazone [10]. For those whose sputum culture does not convert to negative within 6 months of treatment with second-line drugs, DST is repeated. For the purposes of the present analysis, patients in whom at least one of the isolates collected prior to surgery was resistant to at least isoniazid, rifampicin and ofloxacin, plus kanamycin or capreomycin, were defined as having XDR-TB.

At the time of registration for treatment, all MDR- and XDR-TB patients were classified into one of three categories (I: new patient, never treated for TB; II: previously treated for drugsusceptible TB; III: previously treated for MDR- or XDR-TB). All patients were assigned to one of five mutually exclusive treatment outcomes (i.e. cured, completed, default, failure or died), which were defined according to standard internationally accepted definitions [11]. Cured patients were consistently culture-negative during the last 12 months of treatment. Patients were treated for $\geqslant 12$ months following surgery before being defined as failure.

Demographic and risk-factor information, including age, sex, alcohol use, history of previous TB treatment, DST patterns before and during treatment, chest radiography results, data on surgical treatment and final treatment outcome, were obtained from patient records. Proportions were compared using the Chi-squared test and Fisher's exact test, means compared using an unpaired t-test, and trends analysed using the Chi-squared test for linear trend.

The Centers for Disease Control and Prevention (Atlanta, GA, USA) reviewed the protocol for the current project and determined that the present study was part of a public health programme evaluation and did not constitute research on human subjects.

\section{RESULTS}

Of a total of 820 patients who were registered for MDR-TB treatment in Latvia during 2000-2003, 66 (8\%) had XDR-TB, and $17(26 \%)$ of these were treated with adjunctive resectional lung surgery (table 1$)$. Of these, $14(82 \%)$ were male. The patients' ages ranged 27-55 yrs (median $42 \mathrm{yrs}$ ). One (6\%) patient had no history of previous TB treatment, 10 (59\%) had been previously treated for drug-susceptible TB and six (35\%) had been previously treated for MDR-TB. The number of drugs to which the strain of TB was resistant ranged 7-11, with a mean of 9.2. Of the 17 patients, $11(65 \%)$ showed resistance to a fluoroquinolone (ofloxacin) plus both injectable drugs, kanamycin and capreomycin, whereas the six remaining patients were resistant to just one of the injectable drugs. Of the patients, $14(82 \%)$ were tested for HIV infection, and none were HIV-infected.

The median duration of TB treatment prior to surgery was 12 months (range 2-20 months). On chest radiography, 12 $(71 \%)$ patients exhibited unilateral cavities and five (29\%) bilateral cavities. Sixteen (94\%) patients were sputum-culturepositive at the time of surgery, including 10 (59\%) who were sputum-smear-positive. For $15(88 \%)$ patients, the indication for surgery was failure of medical treatment to convert sputum cultures to negative. One patient (No. 15; table 1) underwent surgery due to a large cavity despite sputum conversion. Another patient (No. 11) was treated with surgery following 2 months of pharmacological treatment due to significant pulmonary haemorrhage.

Of the 17 total patients, five (29\%) underwent pneumonectomy, four (24\%) lobectomy and eight (47\%) segmental resections. All five patients with bilateral cavities exhibited asymmetric findings on chest radiography; surgery targeted the lung with the more extensive disease in these patients.

Three patients experienced operative complications, one each with recurrent laryngeal nerve damage, acute respiratory distress syndrome and intrathoracic bleeding, but reoperation was not necessary. There were no surgery-related deaths. Medical treatment generally included drugs to which the isolate had demonstrated susceptibility (table 1). In many cases, injectable drugs and ofloxacin were used despite resistance. Since most patients were resistant to many drugs, leaving few available treatment options, medical treatment was usually not changed following surgery. The median duration of pharmacological treatment for TB following surgery was 14.5 months (range 7-28 months).

Overall, eight (47\%) patients were cured, one $(6 \%)$ died and eight $(47 \%)$ patients failed. Treatment outcomes varied by registration category: one $(100 \%)$ out of one patients with no previous TB treatment were cured, compared to six (60\%) out of 10 patients previously treated for drug-susceptible TB and one $(16 \%)$ out of six patients previously treated for MDR-TB $(p=0.05$; Chi-squared test for trend). Among those who had been treated for MDR-TB previously, four had failed previously, one had defaulted and one had been cured but had had an MDR-TB relapse. XDR-TB patients with isolates resistant to both injectable drugs tested (capreomycin and kanamycin) were less commonly cured than those resistant to only one injectable drug, with four $(36 \%)$ out of 11 and four $(67 \%)$ out of six cured in each group, respectively. This difference was nonsignificant $(p=0.23)$. Isolates from patients who were cured were resistant to a mean of 8.6 drugs, compared to 9.7 drugs in persons who died or failed $(p=0.07)$. Only one $(20 \%)$ out of five patients with bilateral cavities was cured, compared to seven $(58 \%)$ out of 12 of those with unilateral disease $(p=0.18)$.

\section{DISCUSSION}

In the present series of 17 patients treated with adjunctive resectional lung surgery for XDR-TB in Latvia, cure rates are reported that are higher than those previously reported for XDR-TB treatment in Europe [2, 3].

Compared to previous studies of surgery, the present series of patients shows similar chest radiographic findings and a similar duration of therapy following surgery [12-14]. Previous studies report a longer duration of medical therapy before surgery, with medians ranging from 14 months to several years. Patients in the present series have other characteristics associated with worse expected outcomes compared to previous studies. Other studies of surgery for MDR-TB categorised patients according to their indication for surgical 


\begin{tabular}{|c|c|c|c|c|c|c|c|c|c|c|c|c|c|c|c|c|c|c|c|}
\hline  & 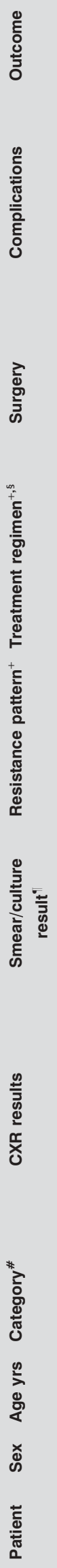 & 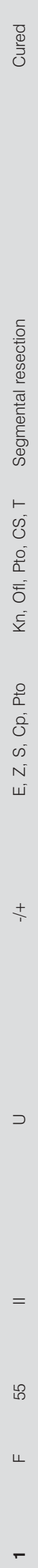 & 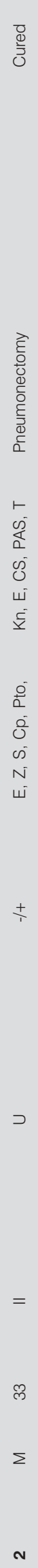 & 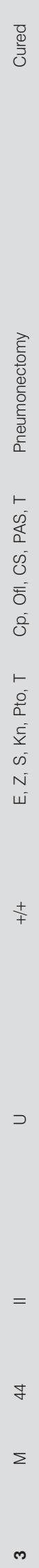 & 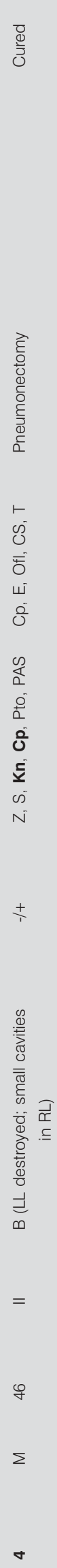 & 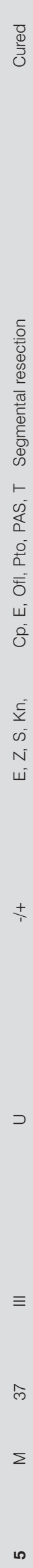 & 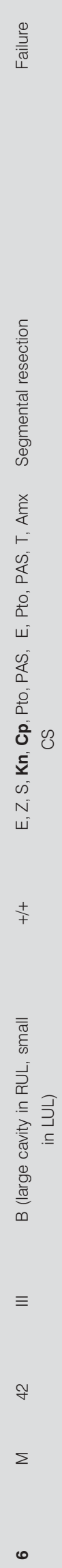 & 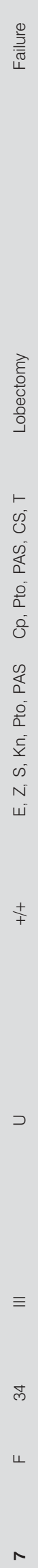 & 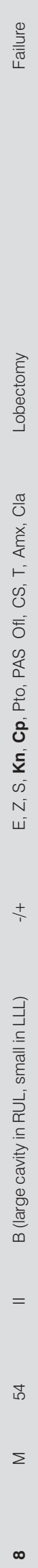 & 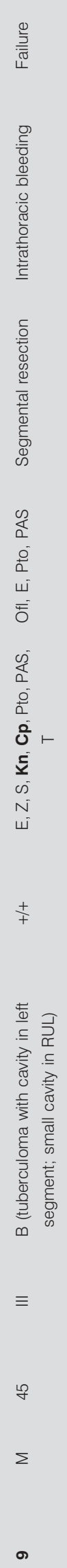 & 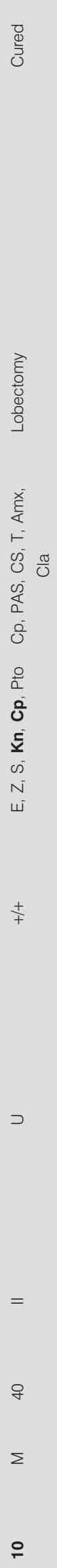 & 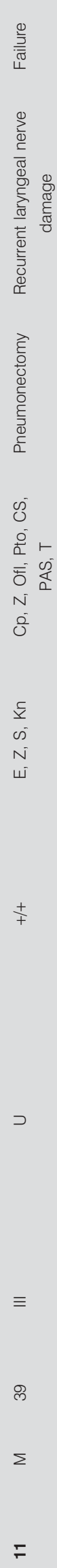 & $\begin{array}{l}0 \\
\text { ㅁ } \\
\delta \\
0 \\
5 \\
\text { s } \\
N \\
\text { w }\end{array}$ & 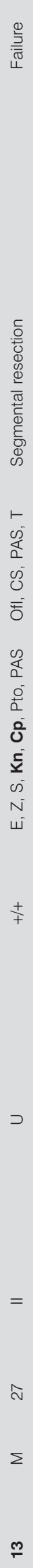 & 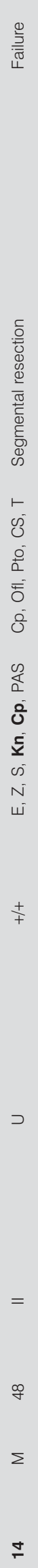 & 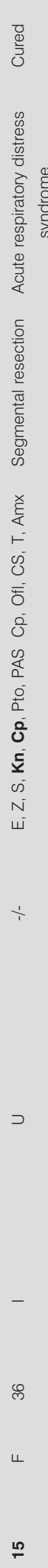 & 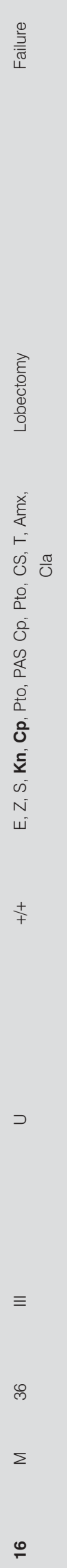 & 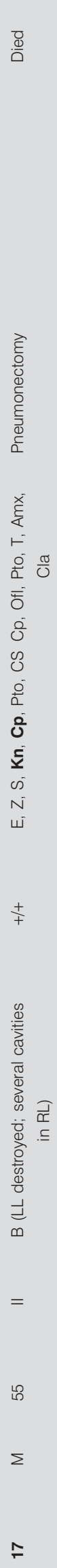 & 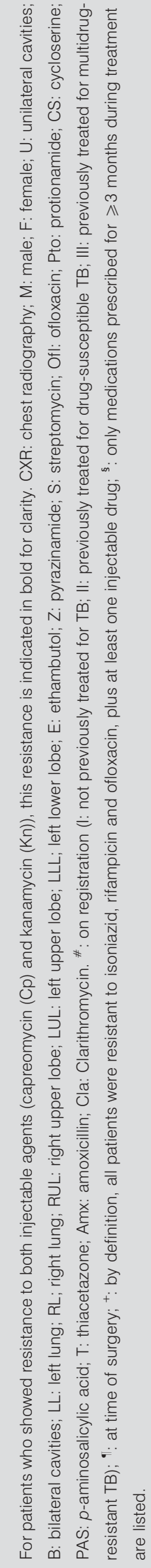 \\
\hline
\end{tabular}


treatment [7]. Since most patients in the present study had the same indication for surgery (failure of medical treatment), it is not possible to compare treatment outcomes based on indication for surgery. However, patients failing medical treatment are less commonly cured than those treated with surgery for other indications [7]. Patients in the present series were generally resistant to many drugs, beyond even those required for definition of $\mathrm{XDR}-\mathrm{TB}$, and all but one of the patients showed resistance to all first-line drugs, decreasing the likelihood of successful treatment [3].

Compared to patients with no prior history of TB treatment, there was a trend towards worse treatment outcomes for patients treated for drug-susceptible $\mathrm{TB}$, and worse outcomes yet for those previously treated for MDR-TB. It is likely that this is because patients treated previously more often exhibited resistance to more drugs, and, in some cases, had not tolerated some drugs in the past, making medical treatment more challenging. There was also a trend towards worse outcomes in patients with resistance to both of the injectable agents, patients with resistance to more drugs and patients with bilateral cavities.

The sample size of the present study was too small to draw specific conclusions regarding factors that may be associated with treatment outcome. Additionally, the case-series nature of the study does not permit comparison of surgery with other treatment options. The present comparison of patient characteristics in the current series to those of patients in previous studies is, in theory, suboptimal since those previous studies were of patients with MDR-TB. Since there are no published series of patients with XDR-TB treated with surgery, this was the only option. In spite of these limitations, the finding that treatment outcomes among this high-risk cohort remained better than those previously reported for XDR-TB patients is an encouraging sign that surgery may be a useful adjunctive treatment in XDR-TB.

With the worldwide emergence of XDR-TB, there is concern that a post-antibiotic phase of TB control is being entered, where there are no longer the drugs necessary to effectively treat persons with drug-resistant TB [15]. More effective treatment, including new drugs for $\mathrm{TB}$, is ultimately essential for the future of XDR-TB treatment. In the interim, efforts should be made to study currently available treatments that have the potential to improve treatment outcomes in these persons. The present data suggest that surgical intervention may constitute a good adjunctive treatment in XDR-TB.

\section{SUPPORT STATEMENT}

The present analysis was funded by the Division of Tuberculosis Elimination of the US Centers for Disease Control and Prevention (Atlanta, GA, USA).

\section{STATEMENT OF INTEREST}

None declared.

\section{ACKNOWLEDGEMENTS}

The authors thank E. McCray and P. LoBue (Division of Tuberculosis Elimination, Centers for Disease Control and Prevention, Atlanta, GA, USA) for their review of the present manuscript.

\section{REFERENCES}

1 Case definition for extensively drug-resistant tuberculosis. Wkly Epidemiol Rec 2006; 81: 408.

2 Sture IS. Treatment outcome among patients with extensively drug-resistant tuberculosis, Latvia, 2000-2004. Int J Tuberc Lung Dis 2007; 11: Suppl. 1, S128.

3 Migliori GB, Besozzi G, Girardi E, et al. Clinical and operational value of the extensively drug-resistant tuberculosis definition. Eur Respir J 2007; 30: 623-626.

4 Shah NS, Wright A, Bai G-H, et al. Worldwide emergence of extensively drug-resistant tuberculosis. Emerg Infect Dis 2007; 13: 380-387.

5 Nathanson E, Lambregts-van Weezenbeek C, Rich ML, et al. Multidrug-resistant tuberculosis management in resource-limited settings. Emerg Infect Dis 2006; 12: 1389-1397.

6 Herzog H. History of tuberculosis. Respiration 1998; 65: 5-15.

7 Chiang C-Y, Yu MC, Bai KJ, et al. Pulmonary resection in the treatment of patients with pulmonary multidrug-resistant tuberculosis in Taiwan. Int J Tuberc Lung Dis 2001; 5: 272-277.

8 Iseman MD, Madsen L, Goble M, et al. Surgical intervention in the treatment of pulmonary disease caused by drug-resistant Mycobacterium tuberculosis. Am Rev Respir Dis 1990; 141: 623-625.

9 Leimane V, Leimans J. Tuberculosis control in Latvia: integrated DOTS and DOTS-plus programmes. Euro Surveill 2006; 11: 29-33.

10 Leimane V, Riekstina V, Holtz TH, et al. Clinical outcome of individualised treatment of multidrug-resistant tuberculosis in Latvia: a retrospective cohort study. Lancet 2005; 365: 318-326.

11 Laserson KF, Thorpe LE, Leimane V, et al. Speaking the same language: treatment outcome definitions for multidrug-resistant tuberculosis. Int J Tuberc Lung Dis 2005; 9: 640-645.

12 Chan ED, Laurel V, Strand MJ, et al. Treatment and outcome analysis of 205 patients with multidrug-resistant tuberculosis. Am J Respir Crit Care Med 2004; 169: 1103-1109.

13 Kim HJ, Kang CH, Kim YT, et al. Prognostic factors for surgical resection in patients with multidrug-resistant tuberculosis. Eur Respir J 2006; 28: 576-580.

14 Takeda S, Maeda H, Hayakawa M, et al. Current surgical intervention for pulmonary tuberculosis. Ann Thorac Surg 2005; 79: 959-963.

15 Raviglione M. XDR-TB: entering the post-antibiotic era? Int J Tuberc Lung Dis 2006; 10: 1185-1187. 